\title{
Character Association and Path Analysis in Bottle Gourd (Lagenaria siceraria L.)
}

\author{
B. Sailaja ${ }^{1 *}$, A. Mohanty ${ }^{2}$, P. Ranjith ${ }^{3}$, G. S. Sahu ${ }^{1}$, D. Lenka ${ }^{4}$ and P. Tripathy ${ }^{1}$ \\ ${ }^{1}$ Department of Vegetable Science, ${ }^{2}$ AICRP on Vegetable Crops, ${ }^{4}$ Department of Plant \\ Breeding and Genetics, College of Agriculture, OUAT, Bhubaneswar, Odisha, India \\ ${ }^{3}$ Department of Plant Breeding and Genetics, College of Horticulture, OUAT, Chiplima, \\ Odisha, India
}

*Corresponding author

\section{A B S T R A C T}

\begin{tabular}{|l|}
\hline Ke y w o r d s \\
Bottle gourd, \\
Character \\
association, Path \\
analysis, Yield \\
attributing \\
characters
\end{tabular}

A field experiment was conducted at All India Co-ordinated Research Project on Vegetable Crops, Odisha University of Agriculture and Technology, Bhubaneswar with seventeen genotypes of bottle gourd [Lagenaria siceraria] in Randomized Block Design with three replications during Post Rabi 2018-19. The result on phenotypic and genotypic correlation coefficient revealed that fruit yield per plant was significantly and positively correlated with fruit weight $(0.799$ and 0.856$)$, node at which female flower appear $(0.310$ and 0.395$)$, leaf area $(0.288$ and 0.357$)$. However, node at which male flower appear (0.085 and -0.109 ) was significantly and negatively correlated with fruit yield per plant at both phenotypic and genotypic level. Further, path coefficient analysis partitioned the correlation into direct and indirect effects. Path analysis revealed that maximum positive direct effect on fruit yield per plant was exhibited through fruit weight, fruit length, days to flowering, 50\% flowering and leaf area, fruit girth, leaf weight, seeds per fruit and fruit circumference these characters play a major role in recombination breeding and suggested that direct selection based on these traits will be rewarded for crop improvement of bottle gourd.

\section{Introduction}

Bottle gourd (Lagenaria siceraria) was domesticated in Asia and at the same time indigenous to Africa (Whitaker and Davis, 1962). The freshly extracted juice of bottle gourd has a tremendous medicinal property that is used for excessive thirst due to severe diarrhea, diabetes and is used in the treatment of epilepsy, stomach acidity, indigestion, ulcers as well as other nervous diseases
(Warrier et al., 1995). In addition to this, the fiber portion helps in preventing constipation and other digestive disorders like flatulence and piles. Bottle gourd is known to lower cholesterol, triglyceride, low-density lipoproteins, pain and inflammation (Ghule et al., 2006).

Yield is a complex trait influenced by genetic factors interacting with environment and is dependent on many other attributes. Other 
yield attributing traits like vine length, node at which first female flower appeared, number of fruits per plant, fruit length, fruit weight etc. are inherited quantitatively and their expression is governed by polygenes which are highly influenced by environment and are less heritable. So selection based on yield components rather than yield itself is reliable and may be accomplished through the component approach of breeding.

A specific difference in environment may have a great effect on some genotypes than others (Shaikh et al., 2012).So, keeping in view of the above facts, the field experiment was designed to study the character association and path analysis of seventeen genotypes of bottle gourd.

\section{Materials and Methods}

The experiment was carried out at All India Co-ordinated Research Project on Vegetable Crops, OUAT, Bhubaneswar, during rabi, 2018-19 with 17 bottle gourd genotypes. The experiment was laid out in Randomized Block Design (RBD) with three replications. In each replication, each entry was grown in a plot having four basins with 3 plants basin ${ }^{-1}$.

From randomly selected 5 plants per each plot observations were recorded for fifteen characters viz., plant height $(\mathrm{cm})$, internodal length $(\mathrm{cm})$, leaf area $\left(\mathrm{m}^{2}\right)$, leaf weight $(\mathrm{kg})$, days to flowering, days to $50 \%$ flowering, node at which female flower appear, node at which male flower appear, fruit length $(\mathrm{cm})$, fruit girth $(\mathrm{cm})$, fruit circumference $(\mathrm{cm})$, fruit weight(g), number of seeds fruit-1, fruit yield plant-1 (g) and fruit yield hectare-1 (q).The data were statistically analysed for computation of character association according to the method suggested by AlJibouri et al., (1958) and path analysis as per Dewey and Lu (1959).

\section{Results and Discussion}

\section{Correlation of fruit yield per plant with other yield components}

According to the results of the present investigation, it was found that the genotypic correlation coefficients were higher than the respective phenotypic correlation coefficients as presented in Table 1 and 2, indicating a strong inherent association among the various characters studied. the yield per plant exhibited highly significant positive correlation with fruit weight, leaf weight, at both phenotypic and genotypic level. These results are in accordance with the findings of (Mahapatra et al., 2019).

These findings indicate the importance of these traits in selection for simultaneous improvement of aforesaid traits along with fruit yield per plant in bottle gourd. Low positive correlation with yield per plant was observed with days to flowering at phenotypic level, internodal length at genotypic level and fruit length at both phenotypic and genotypic level. Similar trend was observed by (Ara et al., 2014) and (Dora et al., 2003).

Some attributes like intermodal length, inflorescence length, fresh weight of root showed negative correlation with yield per plant suggesting that these characters will be taken into consideration for improvement of dolichos bean for earliness.

Traits like days to first flowering and days to $50 \%$ flowering exhibited negative significant correlation with pod yield per plant at both genotypic and phenotypic level which implies least importance for effective selection based on these characters. These results are in agreement with Chaitanya et al., (2014) and Verma et al., (2015). 
Table.1 Phenotypic correlation coefficient $\left(r_{p}\right)$ between all pairs of 14 characters studied in bottle gourd genotypes

\begin{tabular}{|c|c|c|c|c|c|c|c|c|c|c|c|c|c|c|}
\hline & $\begin{array}{c}\text { Plant } \\
\text { height }(\mathrm{cm})\end{array}$ & $\begin{array}{l}\text { Fruit length } \\
\quad(\mathrm{cm})\end{array}$ & $\begin{array}{c}\text { Fruit } \\
\text { circumfe } \\
\text { rence }(\mathrm{cm})\end{array}$ & $\begin{array}{l}\text { Fruit girth } \\
\quad(\mathrm{cm})\end{array}$ & $\begin{array}{c}\text { Leaf } \\
\operatorname{area}\left(\mathbf{m}^{2}\right)\end{array}$ & $\begin{array}{c}\text { Leaf } \\
\text { weight } \\
(\mathrm{kg})\end{array}$ & $\begin{array}{l}\text { Intern } \\
\text { odal } \\
\text { length } \\
(\mathrm{cm})\end{array}$ & $\begin{array}{c}\text { Fruit } \\
\text { weight } \\
\text { (kg) }\end{array}$ & $\begin{array}{c}\text { Seeds/ } \\
\text { fruit }\end{array}$ & $\begin{array}{c}\text { Days to } \\
\text { flowering }\end{array}$ & $\begin{array}{c}\text { Days to } \\
50 \% \\
\text { flowering }\end{array}$ & $\begin{array}{l}\text { Node at } \\
\text { which } \\
\text { female } \\
\text { flower }\end{array}$ & $\begin{array}{l}\text { Node at which } \\
\text { male flower } \\
\text { appear }\end{array}$ & $\begin{array}{c}\text { Yield/ } \\
\text { plant } \\
(\mathbf{k g})\end{array}$ \\
\hline Plant height (cm) & 1.00 & & & & & & & & & & & & & \\
\hline Fruit length $(\mathrm{cm})$ & $-0.453^{* *}$ & 1.00 & & & & & & & & & & & & \\
\hline Fruit circumference $\quad(\mathbf{c m})$ & $0.386^{* *}$ & $-0.816^{* *}$ & 1.00 & & & & & & & & & & & \\
\hline Fruit girth $(\mathbf{c m})$ & $0.385^{* *}$ & $-0.782^{* *}$ & $0.934^{* *}$ & 1.00 & & & & & & & & & & \\
\hline Leaf area $\left(\mathbf{m}^{2}\right)$ & -0.101 & -0.048 & 0.142 & 0.143 & 1.00 & & & & & & & & & \\
\hline Leaf weight (kg) & $-0.288^{*}$ & -0.010 & $0.303^{*}$ & $0.297^{*}$ & $0.700^{* *}$ & 1.00 & & & & & & & & \\
\hline Internodal length $(\mathrm{cm})$ & 0.124 & $0.291^{*}$ & $-0.333^{*}$ & $-0.306^{*}$ & 0.164 & -0.169 & 1.00 & & & & & & & \\
\hline Fruit weight (kg) & $0.340^{*}$ & 0.042 & 0.104 & 0.014 & 0.154 & 0.164 & 0.125 & 1.00 & & & & & & \\
\hline Seeds/fruit & -0.268 & -0.039 & 0.183 & 0.215 & 0.248 & $0.445^{* *}$ & $-\overline{-}^{* * *}$ & 0.036 & 1.00 & & & & & \\
\hline Days to flowering & -0.230 & 0.101 & -0.127 & -0.094 & $0.408^{* *}$ & $0.389^{* *}$ & 0.003 & 0.120 & -0.006 & 1.00 & & & & \\
\hline Days to $50 \%$ flowering & -0.011 & -0.211 & $0.376^{* *}$ & $0.355^{* *}$ & 0.114 & 0.255 & $-0.281^{*}$ & -0.171 & 0.247 & 0.089 & 1.00 & & & \\
\hline $\begin{array}{l}\text { Node at which female flower } \\
\text { Appear }\end{array}$ & 0.156 & -0.081 & 0.126 & 0.214 & 0.103 & 0.082 & 0.187 & 0.076 & 0.147 & 0.009 & $0.379^{* *}$ & 1.00 & & \\
\hline $\begin{array}{l}\text { Node at which male flower } \\
\text { appear }\end{array}$ & 0.132 & $-0.350^{*}$ & $0.275^{*}$ & $0.340^{*}$ & -0.034 & -0.055 & -0.174 & -0.268 & 0.141 & -0.088 & $0.299^{*}$ & $0.528^{* *}$ & 1.00 & \\
\hline Yield/plant (kg) & 0.196 & 0.097 & 0.162 & 0.113 & $0.288^{*}$ & $0.356^{* *}$ & 0.058 & $0.799^{* *}$ & 0.240 & 0.091 & 0.127 & $0.310^{*}$ & -0.085 & 1.00 \\
\hline
\end{tabular}

*Significance level @ 5\% and ** Significance level @ 1\% 


\section{Int.J.Curr.Microbiol.App.Sci (2020) 9(11): 3349-3356}

Table.2 Genotypic correlation coefficient $\left(r_{\mathrm{g}}\right)$ between all pairs of 14 characters studied in bottle gourd genotypes

\begin{tabular}{|c|c|c|c|c|c|c|c|c|c|c|c|c|c|c|}
\hline & $\begin{array}{l}\text { Plant height } \\
\text { (cm) }\end{array}$ & $\begin{array}{l}\text { Fruit } \\
\text { length } \\
(\mathrm{cm})\end{array}$ & $\begin{array}{c}\text { Fruit } \\
\text { circumfe } \\
\text { rence } \\
(\mathbf{c m})\end{array}$ & $\begin{array}{l}\text { Fruit girth } \\
\text { (cm) }\end{array}$ & $\begin{array}{ll}\text { Leaf } & \text { area } \\
(\mathrm{m} 2) & \end{array}$ & $\begin{array}{c}\text { Leaf } \\
\text { weight } \\
(\mathbf{k g})\end{array}$ & $\begin{array}{c}\text { Internod } \\
\text { al length } \\
(\mathbf{c m})\end{array}$ & $\begin{array}{c}\text { Fruit } \\
\text { weight } \\
(\mathbf{k g})\end{array}$ & $\begin{array}{l}\text { Seeds/ } \\
\text { fruit }\end{array}$ & $\begin{array}{l}\text { Days to } \\
\text { flowering }\end{array}$ & $\begin{array}{l}\text { Days to } 50 \% \\
\text { flowering }\end{array}$ & $\begin{array}{c}\text { Node at } \\
\text { which } \\
\text { female } \\
\text { flower } \\
\text { appear }\end{array}$ & $\begin{array}{c}\text { Node at which } \\
\text { male flower } \\
\text { appear }\end{array}$ & $\begin{array}{l}\text { Yield/plant } \\
\text { (kg) }\end{array}$ \\
\hline Plant height (cm) & 1.00 & & & & & & & & & & & & & \\
\hline Fruit length $(\mathrm{cm})$ & $-0.467 * *$ & 1.00 & & & & & & & & & & & & \\
\hline Fruit circumference $(\mathbf{c m})$ & $0.401 * *$ & $-0.837^{* * *}$ & 1.00 & & & & & & & & & & & \\
\hline Fruit girth (cm) & $0.409^{* *}$ & $-0.814 * *$ & $0.964 * *$ & 1.00 & & & & & & & & & & \\
\hline Leaf area $(\mathrm{m} 2)$ & -0.118 & -0.046 & 0.142 & 0.134 & 1.00 & & & & & & & & & \\
\hline Leaf weight (kg) & $-0.326 *$ & -0.008 & 0.320 & 0.300 & $0.787 * *$ & 1.00 & & & & & & & & \\
\hline Internodal length $(\mathbf{c m})$ & 0.112 & $0.307^{*}$ & $-0.383 * *$ & $-0.362 * *$ & 0.167 & -0.252 & 1.00 & & & & & & & \\
\hline Fruit weight (kg) & 0.352 & 0.042 & 0.106 & 0.013 & 0.181 & 0.172 & 0.144 & 1.00 & & & & & & \\
\hline Seeds/fruit & $-0.293 *$ & -0.025 & 0.228 & 0.254 & $0.327 *$ & $0.516^{* *}$ & $-0.512^{* * *}$ & 0.040 & 1.00 & & & & & \\
\hline Days to flowering & $-0.372 * *$ & 0.208 & -0.259 & -0.206 & $0.711 * *$ & $0.680 * *$ & 0.123 & 0.189 & 0.015 & 1.00 & & & & \\
\hline Days to 50\% flowering & 0.029 & $-0.291 *$ & $0.479 * *$ & $0.499 * *$ & 0.146 & $0.433 * *$ & $-0.311^{*}$ & -0.215 & $0.454 * *$ & 0.036 & 1.00 & & & \\
\hline $\begin{array}{l}\text { Node at which female flower } \\
\text { Appear }\end{array}$ & 0.208 & -0.079 & 0.133 & $0.290^{*}$ & 0.160 & 0.156 & 0.240 & 0.090 & 0.198 & -0.038 & $0.499 * *$ & 1.00 & & \\
\hline $\begin{array}{l}\text { Node at which male flower } \\
\text { Appear }\end{array}$ & 0.235 & $-0.524 * *$ & $0.392^{* *}$ & $0.570^{* *}$ & -0.110 & -0.081 & $-0.305^{*}$ & $-0.389^{* *}$ & 0.081 & -0.175 & $0.492 * *$ & $0.759^{* *}$ & 1.00 & \\
\hline Yield/plant (kg) & 0.222 & 0.079 & 0.175 & 0.111 & $0.357 * *$ & $0.394 * *$ & 0.030 & $0.856 * *$ & $0.354 * *$ & $0.377^{* * *}$ & 0.173 & $0.395 * *$ & -0.109 & 1.00 \\
\hline
\end{tabular}

*Significance level at $5 \%$ and ** Significance level at $1 \%$ 


\section{Int.J.Curr.Microbiol.App.Sci (2020) 9(11): 3349-3356}

Table.3 Estimate of direct (diagonal) and indirect effect of component characters on yield in bottle gourd genotypes

\begin{tabular}{|c|c|c|c|c|c|c|c|c|c|c|c|c|c|c|}
\hline & $\begin{array}{l}\text { Plant } \\
\text { Height } \\
(\mathbf{c m})\end{array}$ & $\begin{array}{l}\text { Fruit } \\
\text { length } \\
(\mathrm{cm})\end{array}$ & $\begin{array}{c}\text { Fruit } \\
\text { circumference } \\
(\mathbf{c m})\end{array}$ & $\begin{array}{l}\text { Fruit } \\
\text { girth } \\
(\mathrm{cm})\end{array}$ & $\begin{array}{l}\text { Leaf } \\
\text { area } \\
(\mathrm{m} 2)\end{array}$ & $\begin{array}{c}\text { Leaf } \\
\text { Weight } \\
(\mathbf{k g})\end{array}$ & $\begin{array}{c}\text { Internodal } \\
\text { length } \\
(\mathrm{cm})\end{array}$ & $\begin{array}{c}\text { Fruit } \\
\text { weight } \\
(\mathbf{k g})\end{array}$ & $\begin{array}{l}\text { Seeds/ } \\
\text { fruit }\end{array}$ & $\begin{array}{l}\text { Days to } \\
\text { flowering }\end{array}$ & $\begin{array}{l}\text { Days to } \\
\mathbf{5 0 \%} \\
\text { Flowering }\end{array}$ & $\begin{array}{c}\text { Node at } \\
\text { which } \\
\text { Female } \\
\text { flower } \\
\text { appear }\end{array}$ & $\begin{array}{c}\text { Node at } \\
\text { which male } \\
\text { Flower } \\
\text { appear }\end{array}$ & $\begin{array}{l}\text { Yield/ } \\
\text { Plant (kg) }\end{array}$ \\
\hline Plant height (cm) & -0.022 & -0.118 & 0.017 & 0.042 & -0.011 & -0.0151 & -0.004 & 0.272 & -0.013 & 0.023 & -0.001 & 0.024 & 0.004 & 0.196 \\
\hline Fruit length $(\mathrm{cm})$ & 0.009 & 0.262 & -0.037 & -0.086 & -0.005 & -0.0005 & -0.010 & 0.033 & -0.001 & -0.010 & -0.032 & -0.012 & -0.010 & 0.097 \\
\hline Fruit circumference (cm) & -0.008 & -0.213 & 0.046 & 0.103 & 0.016 & 0.015 & 0.011 & 0.082 & 0.009 & 0.012 & 0.057 & 0.019 & 0.008 & 0.162 \\
\hline Fruit girth (cm) & -0.008 & -0.205 & 0.043 & 0.111 & 0.016 & 0.015 & 0.010 & 0.011 & 0.010 & 0.009 & 0.054 & 0.032 & 0.010 & 0.113 \\
\hline Leaf area $(\mathrm{m} 2)$ & 0.002 & -0.012 & 0.006 & 0.015 & 0.118 & 0.036 & -0.005 & 0.123 & 0.012 & -0.040 & 0.017 & 0.015 & -0.001 & $0.288^{*}$ \\
\hline Leaf weight (kg) & 0.006 & -0.002 & 0.013 & 0.032 & 0.082 & 0.053 & 0.005 & 0.130 & 0.022 & -0.039 & 0.039 & 0.012 & -0.001 & $0.356^{* *}$ \\
\hline Internodal length $(\mathbf{c m})$ & -0.002 & 0.076 & -0.015 & -0.033 & 0.019 & -0.008 & -0.035 & 0.100 & -0.021 & -0.0003 & -0.043 & 0.028 & -0.005 & 0.058 \\
\hline Fruit weight (kg) & -0.007 & 0.011 & 0.004 & 0.001 & 0.018 & 0.008 & -0.004 & 0.799 & 0.001 & -0.0120 & -0.026 & 0.011 & -0.008 & $0.799 * *$ \\
\hline Seeds/fruit & 0.005 & -0.010 & 0.008 & 0.023 & 0.029 & 0.023 & 0.014 & 0.028 & 0.051 & 0.0006 & 0.038 & 0.022 & 0.004 & 0.240 \\
\hline Days to flowering & 0.005 & 0.026 & -0.005 & -0.010 & 0.048 & 0.020 & -0.0001 & 0.095 & -0.0003 & -0.1 & 0.013 & 0.001 & -0.002 & 0.091 \\
\hline Days to 50\% flowering & 0.0002 & -0.055 & 0.017 & 0.039 & 0.013 & 0.013 & 0.009 & -0.136 & 0.012 & -0.008 & 0.154 & 0.058 & 0.009 & 0.127 \\
\hline Node at which female flower appear & -0.003 & -0.021 & 0.005 & 0.023 & 0.012 & 0.004 & -0.006 & 0.060 & 0.007 & -0.0008 & 0.058 & 0.154 & 0.016 & $0.310^{*}$ \\
\hline Node at which male flower appear & -0.002 & -0.091 & 0.012 & 0.037 & -0.004 & -0.002 & 0.006 & -0.214 & 0.007 & 0.008 & 0.045 & 0.081 & 0.031 & -0.08 \\
\hline
\end{tabular}

Residual effects: 0.18027 


\section{Int.J.Curr.Microbiol.App.Sci (2020) 9(11): 3349-3356}

Table.4 Estimate of direct (diagonal) and indirect effect of component characters on yield per plant in bottle gourd genotype

\begin{tabular}{|c|c|c|c|c|c|c|c|c|c|c|c|c|c|c|}
\hline & $\begin{array}{r}\text { Plant } \\
\text { height } \\
\text { (cm) }\end{array}$ & $\begin{array}{l}\text { Fruit } \\
\text { length } \\
\text { (cm) }\end{array}$ & $\begin{array}{l}\text { Fruit circumference } \\
(\mathbf{c m})\end{array}$ & $\begin{array}{l}\text { Fruit } \\
\text { girth } \\
(\mathrm{cm})\end{array}$ & $\begin{array}{l}\text { Leaf } \\
\text { area } \\
(\mathrm{m} 2)\end{array}$ & $\begin{array}{c}\text { Leaf } \\
\text { weight } \\
(\mathrm{kg})\end{array}$ & $\begin{array}{c}\text { Internodal } \\
\text { length } \\
(\mathrm{cm})\end{array}$ & $\begin{array}{c}\text { Fruit } \\
\text { weight } \\
(\mathbf{k g})\end{array}$ & $\begin{array}{l}\text { Seeds/ } \\
\text { fruit }\end{array}$ & $\begin{array}{c}\text { Days to } \\
\text { flowering }\end{array}$ & $\begin{array}{c}\text { Days to } 50 \% \\
\text { Flowering }\end{array}$ & $\begin{array}{c}\text { Node at which } \\
\text { female flower } \\
\text { appear }\end{array}$ & $\begin{array}{c}\text { Node at which } \\
\text { male flower } \\
\text { appear }\end{array}$ & Yield/ plant (kg) \\
\hline Plant height (cm) & -0.908 & -0.481 & -0.272 & 0.606 & -0.213 & 0.637 & -0.065 & 0.637 & 0.153 & 0.094 & 0.033 & -0.102 & 0.103 & 0.222 \\
\hline Fruit length (cm) & 0.424 & 1.03 & 0.568 & -1.207 & -0.083 & 0.016 & -0.180 & 0.076 & 0.013 & -0.052 & -0.334 & 0.039 & -0.230 & 0.079 \\
\hline Fruit circumference (cm) & -0.364 & -0.862 & -0.679 & 1.429 & 0.258 & -0.625 & 0.225 & 0.190 & -0.119 & 0.065 & 0.550 & -0.065 & 0.172 & 0.175 \\
\hline Fruit girth $(\mathbf{c m})$ & -0.371 & -0.838 & -0.654 & 1.483 & 0.242 & -0.585 & 0.213 & 0.023 & -0.133 & 0.052 & 0.573 & -0.143 & 0.250 & 0.111 \\
\hline Leaf area $(\mathbf{m} 2)$ & 0.106 & -0.047 & -0.096 & 0.198 & 1.817 & -1.539 & -0.098 & 0.327 & -0.171 & -0.180 & 0.167 & -0.078 & -0.048 & $0.357^{* *}$ \\
\hline Leaf weight (kg) & 0.296 & -0.008 & -0.217 & 0.444 & 1.430 & -1.955 & 0.148 & 0.311 & -0.270 & -0.172 & 0.498 & -0.077 & -0.035 & $0.394 * *$ \\
\hline Internodal length (cm) & -0.101 & 0.316 & 0.260 & -0.537 & 0.303 & 0.491 & -0.589 & 0.259 & 0.268 & -0.031 & -0.357 & -0.118 & -0.133 & 0.030 \\
\hline Fruit weight $(\mathbf{k g})$ & -0.319 & 0.043 & -0.071 & 0.019 & 0.329 & -0.336 & -0.084 & 1.809 & -0.021 & -0.047 & -0.247 & -0.044 & -0.170 & 0.856 ** \\
\hline Seeds/fruit & 0.266 & -0.025 & -0.155 & 0.377 & 0.593 & -1.008 & 0.301 & 0.072 & -0.524 & -0.003 & 0.522 & -0.098 & 0.035 & $0.354 * *$ \\
\hline Days to flowering & 0.337 & 0.213 & 0.175 & -0.305 & 1.292 & -1.328 & -0.072 & 0.341 & -0.008 & -0.254 & 0.041 & 0.018 & -0.077 & $0.377 * *$ \\
\hline Days to $50 \%$ flowering & -0.026 & -0.299 & -0.325 & 0.740 & 0.264 & -0.847 & 0.183 & -0.388 & -0.237 & -0.009 & 1.15 & -0.246 & 0.216 & 0.173 \\
\hline Node at which female flower appear & -0.188 & -0.081 & -0.090 & 0.430 & 0.289 & -0.304 & -0.141 & 0.163 & -0.103 & 0.009 & 0.573 & -0.494 & 0.333 & $0.395 * *$ \\
\hline Node at which male flower appear & -0.213 & $\begin{array}{c}- \\
0.5397\end{array}$ & -0.266 & 0.844 & -0.200 & 0.158 & 0.179 & -0.703 & -0.042 & 0.044 & 0.566 & -0.375 & 0.439 & -0.109 \\
\hline
\end{tabular}


From the above discussion on correlation it may be inferred that with fruit weight, leaf weight, node at which female flower \& male flower appear are the important correlated characters effect on yield per plant and simultaneous improvement in these characters will be helpful in bottle gourd improvement programme (Table 3 and 4).

Correlation coefficient which measures the association between any two characters may not give a true comprehensive picture in a complex situation which creates a difficulty in deciding the breeding procedure to be adopted or making simultaneous selection for crop improvement.

Path analysis helps in identifying the most promising components and minimises undesired characters in selection process through partitioning the total correlation into direct and indirect effects of different components.

In the present study as per the data presented in Table 4, it was found that fruit weight showed highly significant phenotypic and genotypic correlation with yield per plant. It exerted maximum direct positive direct effect on yield per plant and indirect positive effect via dependent variable like fruit length, node at which female flower appear, leaf area, seeds per fruit, leaf weight and days to flowering. This suggested that direct selection based on these traits would be rewarding for crop improvement. These findings are in agreement with the observations of (Raut et al., 2013 and Pandey et al., 2012).

Negative correlation of node at which male flower appears with yield per plant is mainly due to negative effect of plant height, fruit length, fruit weight and leaf area. The same findings are in agreement with the observation of (Deepti et al., 2013 and Janaranjan et al., 2015).
From the correlation and path analysis it can be concluded that it can be concluded that the traits like plant height, fruit circumference, fruit length, fruit weight, leaf weight, seeds per fruit, $50 \%$ flowering exhibited positive direct effects on fruit yield per plant and selection based on these characters will result in the development of high yielding superior genotypes of bottle gourd. Therefore these characters rewarding to give more focus while selection of genotypes for increasing yield of bottle gourd crop.

\section{References}

Ara Z, Zakaria M, Uddin MZ, Rahman MM, Rasul MG, Kabir AFMR. 2014. Correlation matrix among different parameters of bottle gourd genotypes. International Journal of Natural and Social Sciences. 1:48-51.

Dora, D. K., Behera, T. K., Acharya, G.C., Mohapatra, P. and Mishra, B. 2003. Genetic variability and character association in pointed gourd (Trichosanthes dioica roxb.). Indian Journal of Horticulture, 60(2): 163-166.

Ghule BV, Ghante MH, Saoji AN, Yeole PG. 2006. Hypolipidermic and Antihyperlipidermic effects of Lagenaria siceraria (mol.) Fruit extracts. Indian journal of experimental biology, 44(11): 905-909.

Mahapatra.S, Sureja. AK, Behera. TK and Bhardwaj. R. 2019.Correlation and path coefficient analyses of quantitative traits in bottle gourd [Lagenaria siceraria (mol.) Standl.] Genotypes. International journal of chemical studies 2019; 7(3): 827-830.

Raut RL, Mishra SP, Verma A and Jain PK. 2013. Correlation studies in bottle gourd, JNKVV Research Journal, 47(1): 76-79.

Shaikh JA, Kathiria KB and Acharya RR.2012. Stability analysis for fruit yield and its component traits in bottle 
gourd, vegetable science, 39(1): 89-91. Whitaker, T.W., Davis, G.N. Cucurbits:

York: Interscience, 1962. 250p. botany, cultivation and utilization. New

\section{How to cite this article:}

Sailaja, B., A. Mohanty, P. Ranjith, G. S. Sahu, D. Lenka and Tripathy, P. 2020. Character Association and Path Analysis in Bottle Gourd (Lagenaria siceraria L.). Int.J.Curr.Microbiol.App.Sci. 9(11): 3349-3356. doi: https://doi.org/10.20546/ijcmas.2020.911.400 